\title{
EIGENVALUES OF SCHRÖDINGER OPERATORS ON FINITE AND INFINITE INTERVALS
}

\author{
EVGENY L. KOROTYAEV
}

\begin{abstract}
We consider a Sturm-Liouville operator a with integrable potential $q$ on the unit interval $I=[0,1]$. We consider a Schrödinger operator with a real compactly supported potential on the half line and on the line, where this potential coincides with $q$ on the unit interval and vanishes outside $I$. We determine the relationships between eigenvalues of such operators and obtain estimates of eigenvalues in terms of potentials.
\end{abstract}

\section{INTRODUCTION AND MAIN RESULTS}

In this paper we discuss eigenvalues of Schrödinger operators defined by

$$
\begin{aligned}
& \text { Case 1: } \quad T y=-y^{\prime \prime}+q y \quad \text { on } \quad L^{2}\left(\mathbb{R}_{+}\right), \quad \text { with } y(0)=0 \text {, } \\
& \text { Case 2: } \quad \widetilde{T} y=-y^{\prime \prime}+q y \quad \text { on } L^{2}\left(\mathbb{R}_{+}\right), \quad \text { with } y^{\prime}(0)=0 \text {, } \\
& \text { Case 3: } \quad \mathcal{T} y=-y^{\prime \prime}+q y \quad \text { on } \quad L^{2}(\mathbb{R}),
\end{aligned}
$$

and defined by (1.3)-(1.5) on the unit interval. We assume that the potential $q$ satisfies

$$
\operatorname{supp} q \in[0,1], \quad q \in L_{\text {real }}^{1}(0,1) .
$$

It is well known ([9], [8], 23]) that their spectrum consists of an absolutely continuous part $[0, \infty)$ plus a finite number of simple negative eigenvalues given by

$$
\begin{gathered}
\sigma_{a c}(T)=[0, \infty), \quad \sigma_{d}(T)=\left\{E_{1}<\cdots<E_{m}<0\right\}, \\
\sigma_{a c}(\widetilde{T})=[0, \infty), \quad \sigma_{d}(\widetilde{T})=\left\{\widetilde{E}_{1}<\cdots<\widetilde{E}_{N}<0\right\}, \\
\sigma_{a c}(\mathcal{T})=[0, \infty), \quad \sigma_{d}(\mathcal{T})=\left\{\mathcal{E}_{1}<\cdots<\mathcal{E}_{\mathcal{N}}<0\right\} .
\end{gathered}
$$

We introduce Sturm-Liouville operators on the interval [0,1] with the Dirichlet and Neumann boundary conditions:

$$
\begin{aligned}
& H_{0} f=-f^{\prime \prime}+q f, \quad f(0)=f(1)=0, \\
& H_{1} f=-f^{\prime \prime}+q f, \quad f^{\prime}(0)=f^{\prime}(1)=0,
\end{aligned}
$$

and with so-called mixed boundary conditions:

$$
\begin{array}{ll}
H_{01} f=-f^{\prime \prime}+q f, & f(0)=f^{\prime}(1)=0, \\
H_{10} f=-f^{\prime \prime}+q f, & f^{\prime}(0)=f(1)=0,
\end{array}
$$

and the operator $H_{\pi}$ on $[0,2]$ with 2-periodic boundary conditions:

$$
H_{\pi} f=-f^{\prime \prime}+q_{\pi} f, \quad q_{\pi}=\left\{\begin{array}{ll}
q(x), & x \in[0,1] \\
q(x-1), & x \in[1,2]
\end{array} .\right.
$$

Date: January 29, 2020.

1991 Mathematics Subject Classification. 34L40 (34L15 34L20).

Key words and phrases. eigenvalues, Sturm-Liuvill problem. 
Let $\mu_{n}$ and $\nu_{0}, \nu_{n}, n \geqslant 1$ be eigenvalues of $H_{0}$ and $H_{1}$ respectively. Let $\tau_{n}$ and $\varrho_{n}, n \geqslant 1$ be eigenvalues of $H_{01}$ and $H_{10}$ respectively. All these eigenvalues are simple. It is well known, that the spectrum of $H_{\pi}$ discrete and its eigenvalues $\lambda_{0}^{+}, \lambda_{n}^{ \pm}, n \geqslant 1$ satisfy $\lambda_{n-1}^{+}<\lambda_{n}^{-} \leqslant \lambda_{n}^{+}, n \geqslant 1$. Moreover, all these eigenvalues satisfy

$$
\begin{aligned}
& \nu_{0} \leqslant \lambda_{0}^{+}<\overline{\varrho_{1}, \tau_{1}}<\lambda_{1}^{-} \leqslant \overline{\mu_{1}, \nu_{1}} \leqslant \lambda_{1}^{+}<\overline{\varrho_{2}, \tau_{2}}<\lambda_{2}^{-} \leqslant \overline{\mu_{2}, \nu_{2}} \leqslant \lambda_{2}^{+}<\ldots, \\
& \lambda_{n}^{ \pm}, \nu_{n}, \mu_{n}=(\pi n)^{2}+c_{0}+o(1), \quad \varrho_{n}, \tau_{n}=\pi^{2}\left(n-\frac{1}{2}\right)^{2}+c_{0}+o(1) \quad \text { as } n \rightarrow \infty,
\end{aligned}
$$

where $\overline{u, v}$ denotes $\min \{u, v\} \leqslant \max \{u, v\}$ for shortness and $c_{0}=\int_{0}^{1} q d x$.

Given a self-adjoint, bounded from below operator $A$ whose negative spectrum is discrete, we denote by $n_{-}(A)$ the number of its negative eigenvalues, counted with multiplicities.

Theorem 1.1. i) The eigenvalues $E_{1}<\cdots<E_{m}<0$ of $T$ for the Case 1 satisfy

$$
\begin{gathered}
n_{-}(T)=n_{-}\left(H_{01}\right) \geqslant n_{-}\left(H_{0}\right), \\
\tau_{1}<E_{1}<\mu_{1}<\tau_{2}<E_{2}<\mu_{2}<\cdots<\tau_{m}<E_{m}<\min \left\{0, \mu_{m}\right\} .
\end{gathered}
$$

ii) The eigenvalues $\widetilde{E}_{1}<\cdots<\widetilde{E}_{N}<0$ of $\widetilde{T}$ for the Case 2 satisfy

$$
n_{-}(\widetilde{T})=n_{-}\left(H_{1}\right) \text {, }
$$

and

$$
\begin{gathered}
\nu_{0}<\widetilde{E}_{1}<\varrho_{1}<\nu_{1}<\widetilde{E}_{2}<\varrho_{2}<\cdots<\nu_{N-1}<\widetilde{E}_{N}<\min \left\{0, \varrho_{N}\right\}, \\
\widetilde{E}_{1}<E_{1}<\widetilde{E}_{2}<E_{2}<\cdots<\widetilde{E}_{N}, \quad\left\{\begin{array}{ll}
\text { if } & \tau_{N} \geqslant 0 \Rightarrow m=N-1 \\
\text { if } & \tau_{N}<0 \Rightarrow m=N \text { and } \widetilde{E}_{N}<E_{N}
\end{array} .\right.
\end{gathered}
$$

iii) The operator $\mathcal{T}$ for the Case 3 has the eigenvalues $\mathcal{E}_{1}<\ldots<\mathcal{E}_{N}$, which satisfy

$$
\begin{gathered}
n_{-}(\mathcal{T})=n_{-}(\widetilde{T})=n_{-}\left(H_{1}\right) \\
\nu_{0}<\widetilde{E}_{1}<\mathcal{E}_{1}<E_{1}<\nu_{1}<\widetilde{E}_{2}<\mathcal{E}_{2}<\cdots<\nu_{N-1}<\widetilde{E}_{N}<\mathcal{E}_{N}<\min \left\{0, E_{N}\right\} .
\end{gathered}
$$

Remark. Thus eigenvalues $\left\{\tau_{n}\right\}$ control $\left\{E_{n}\right\} ;\left\{\nu_{n}\right\}$ control both $\left\{\widetilde{E}_{n}\right\}$ and $\left\{\mathcal{E}_{n}\right\}$.

We describe eigenvalues for $\mathcal{T}_{e} y=-y^{\prime \prime}+q_{e} y$ on $L^{2}(\mathbb{R})$ with even potentials $q_{e}(x)=q(|x|)$ for all $x \in \mathbb{R}$. Using Theorem 1.1 and the known identity (2.23) we obtain

Corollary 1.2. The eigenvalues $\mathcal{E}_{1}^{e}<\cdots<\mathcal{E}_{n_{e}}^{e}<0$ of $\mathcal{T}_{e}$ coincide with eigenvalues of the operators $T$ and $\widetilde{T}$ given by (1.1) and satisfy

$$
\begin{gathered}
\sigma_{d}\left(\mathcal{T}_{e}\right)=\sigma_{d}(T) \cup \sigma_{d}(\widetilde{T}), \quad n_{e}=n_{-}\left(\mathcal{T}_{e}\right)=N+m=\left\{\begin{array}{l}
2 N-1 \text { if } \tau_{N} \geqslant 0 \\
2 N \quad \text { if } \tau_{N}<0
\end{array},\right. \\
\nu_{0}<\mathcal{E}_{1}^{e}<\varrho_{1} ; \quad \tau_{1}<\mathcal{E}_{2}^{e}<\mu_{1} ; \quad \nu_{1}<\mathcal{E}_{3}^{e}<\varrho_{2} ; \quad \tau_{2}<\mathcal{E}_{4}^{e}<\mu_{2} ; \ldots
\end{gathered}
$$

Localization of resonances. We discuss resonances for the class compactly supported potentials $\mathcal{P}=\left\{f \in L_{\text {real }}^{1}\left(\mathbb{R}_{+}\right): \sup \operatorname{supp} f=1\right\}$. We define the Jost solutions $f_{+}(x, k)$ of the equation

$$
-f^{\prime \prime}+q(x) f=k^{2} f, \quad k \in \mathbb{C} \backslash\{0\},
$$

under the conditions: $f_{+}(x, k)=e^{i x k}$ for $x>1$. The Jost function $\psi=f_{+}(0, \cdot)$ is entire and satisfies

$$
\psi(k)=1+O(1 / k) \quad \text { as } \quad|k| \rightarrow \infty, \quad k \in \overline{\mathbb{C}}_{+},
$$


uniformly in $\arg k \in[0, \pi]$. The function $\psi(k)$ has $m$ simple zeros $k_{j}, j \in \mathbb{N}_{m}=\{1, . ., m\}$ in $\mathbb{C}_{+}$, given by $k_{j}=i\left|E_{j}\right|^{\frac{1}{2}}, j \in \mathbb{N}_{m}$, possibly one simple zero at 0 , and an infinite number (so-called resonances) in $\mathbb{C}_{-}: 0 \leqslant\left|k_{m+1}\right| \leqslant\left|k_{m+2}\right| \leqslant \ldots \ldots$. The function $\psi$ has an odd number $\geqslant 1$ of zeros on any interval $I_{j}:=\left(-k_{j},-k_{j+1}\right), j \in \mathbb{N}_{m-1}$ and an even number $\geqslant 0$ of zeros on the interval $I_{m}:=\left(-i\left|k_{m}\right|, 0\right]$ counted with multiplicity. Let $\#(E)$ be the number of zeros of $\psi$ (counted with multiplicity) in the set $E \subset \mathbb{C}$. We describe the localization of resonances on the interval $\left[-k_{1}, 0\right]$ in terms of eigenvalues .

Theorem 1.3. i) Let $q \in \mathcal{P}$ and let a resonance $k_{o} \in I_{j} \subset i \mathbb{R}_{-}$for some $j \in \mathbb{N}_{m}$. Then

$$
\mu_{j}<k_{o}^{2}<\tau_{j+1}, \quad j \in \mathbb{N}_{m}=\{1, . ., m\} .
$$

ii) For any finite sequence $p_{1}, p_{2}, . ., p_{m} \in i \mathbb{R}_{+}$(labeled by $\left|p_{1}\right|>\left|p_{2}\right|>\ldots>\left|p_{m}\right|>0$ ) and any odd integers $s_{1}, \ldots, s_{m-1} \in \mathbb{N}$ and even integer $s_{m} \geqslant 0$ there exists a potential $q \in \mathcal{P}$, such that each its eigenvalue $E_{j}=p_{j}^{2}<0$ and $\#\left(I_{j}\right)=s_{j}, j=\mathbb{N}_{m}$.

Remark. 1) Thus eigenvalues $\mu_{j}, \tau_{j+1}$ control resonances on the interval $I_{j}$.

2) If $\mu_{m} \geqslant 0$, then there are no resonances on the interval $\left(-k_{m}, 0\right]$.

3) Jost and Kohn [14] adapted the method of Gelfand and Levitan to determine a potential (exponentially decaying) of a Schrödinger operator by the spectral data. Theorem 1.3 is used in the paper [21] to show that Jost and Kohn solution is not complete and there exists another solution, which gives a compactly supported potential.

Estimates. Recall the Bargmann inequality [3]: let in general, $x q(x)$ belong to $L^{1}(0, \infty)$, then

$$
n_{-}(T) \leqslant \int_{0}^{\infty} x q_{-}(x) d x
$$

where $q_{-}=\max \{-q, 0\}$. The case of distributions was discussed in [1], and more operators was studied in [2]. Recall the Calogero-Cohn inequality [4], [5]: if $q,|q|^{\frac{1}{2}} \in L^{1}(0, \infty)$ and if $q \leqslant 0$ is monotone, then

$$
n_{-}(T) \leqslant \frac{2}{\pi} \int_{0}^{\infty}|q(x)|^{\frac{1}{2}} d x .
$$

We recall the estimates from [19], 20]: Consider the operator $H_{\pi}$ with the potential $q \in$ $\mathscr{H}_{0}=\left\{q \in L_{\text {real }}^{2}(0,1): \int_{0}^{1} q(x) d x=0\right\}$ having eigenvalues $\lambda_{0}^{+}, \lambda_{n}^{ \pm}, n \geqslant 1$. Let $\gamma_{n}=\lambda_{n}^{+}-\lambda_{n}^{-} \geqslant$ $0, n \geqslant 1$ and $\gamma=\left(\sum_{n \geqslant 1} \gamma_{n}^{2}\right)^{\frac{1}{2}} \geqslant 0$. Then

$$
\begin{array}{r}
\|q\| \leqslant 2 \gamma \max \left\{1, \gamma^{\frac{1}{3}}\right\}, \\
\gamma \leqslant 2\|q\| \max \left\{1,\|q\|^{\frac{1}{3}}\right\} .
\end{array}
$$

Corollary 1.4. Let the operators $H_{0}, H_{01}, H_{\pi}$ given by (1.3)-(1.5).

i) Let $\mathfrak{S}=\sum_{\mu_{n}<0}\left|\mu_{n}\right|^{\frac{1}{2}}$ or $\mathfrak{S}=\frac{1}{2} \sum_{n>0, \lambda_{n}^{ \pm}<0}\left|\lambda_{n}^{ \pm}\right|^{\frac{1}{2}}$. Then

$$
\begin{gathered}
\frac{1}{2}\left(n_{-}\left(H_{\pi}\right)-1\right) \leqslant n_{-}\left(H_{0}\right) \leqslant n_{-}\left(H_{01}\right) \leqslant \int_{0}^{1} x q_{-}(x) d x, \\
\mathfrak{S} \leqslant \frac{1}{2} \int_{0}^{1} q_{-}(x) d x,
\end{gathered}
$$


Let $q(x)$ be monotone. Then

$$
n_{-}\left(H_{0}\right) \leqslant n_{-}\left(H_{01}\right) \leqslant \frac{2}{\pi} \int_{0}^{1} q_{-}(x)^{\frac{1}{2}} d x .
$$

ii) Let $q \in L^{2}(0,1)$ and $\int_{0}^{1} q(x) d x=0$. Then

$$
\begin{aligned}
& \frac{1}{2}\left(n_{-}\left(H_{\pi}\right)-1\right) \leqslant n_{-}\left(H_{0}\right) \leqslant n_{-}\left(H_{01}\right) \leqslant 2 \gamma \max \left\{1, \gamma^{\frac{1}{3}}\right\}, \\
& \mathfrak{S} \leqslant 2 \gamma \max \left\{1, \gamma^{\frac{1}{3}}\right\} .
\end{aligned}
$$

Remark. Instead of $q$ in Corollary 1.4 we can use $q-\varepsilon$ for $\varepsilon \in \mathbb{R}$

There are a lot of results devoted to estimates of negative eigenvalues of $\operatorname{dim} d=1$ Schrödinger operators, see [4], [5], [3], [7], [11], [25], [26] and references therein.

There exist many results about Sturm-Liouville operators on the unit interval, see [6], [12, [22], [23], [24] and on the circle, see [10], [18], [23] and references therein. Unfortunately there only few results about estimates for such Sturm-Liouville operators, for example, two-sided estimates of periodic potentials in terms of gap-lengths see (1.21).

\section{Proof}

2.1. Fundamental solutions. Let $\varphi(x, \lambda), \vartheta(x, \lambda)$ be the solutions of the equation

$$
-y^{\prime \prime}+q y=\lambda y, \quad \lambda \in \mathbb{C},
$$

under the conditions $\varphi^{\prime}(0, \lambda)=\vartheta(0, \lambda)=1$ and $\varphi(0, \lambda)=\vartheta^{\prime}(0, \lambda)=0$. If $q=0$, then the corresponding fundamental solutions are given by $\varphi_{0}(x, \lambda)=\frac{\sin \sqrt{\lambda} x}{\sqrt{\lambda}}$ and $\vartheta_{0}(x, \lambda)=\cos x \sqrt{\lambda}$. Recall the well-known results.

Lemma 2.1. Let $q \in L^{1}(0,1)$. Then the functions $\varphi(1, \lambda), \varphi^{\prime}(1, \lambda), \vartheta(1, \lambda), \vartheta^{\prime}(1, \lambda)$ are entire and satisfy:

$$
\begin{gathered}
\varphi(1, \lambda)-\frac{\sin \sqrt{\lambda}}{\sqrt{\lambda}}=e^{|\operatorname{Im} \sqrt{\lambda}|} O\left(\lambda^{-1}\right), \\
\varphi^{\prime}(1, \lambda)-\cos \sqrt{\lambda}=e^{|\operatorname{Im} \sqrt{\lambda}|} O\left(\lambda^{-\frac{1}{2}}\right),
\end{gathered}
$$

and

$$
\begin{gathered}
\vartheta(1, \lambda)-\cos \sqrt{\lambda}=e^{|\operatorname{Im} \sqrt{\lambda}|} O\left(\lambda^{-\frac{1}{2}}\right), \\
\vartheta^{\prime}(1, z)+\sqrt{\lambda} \sin \sqrt{\lambda}=e^{|\operatorname{Im} \sqrt{\lambda}|} O(1),
\end{gathered}
$$

as $|\lambda| \rightarrow \infty$, uniformly in $\arg \lambda \in[0,2 \pi]$, and, in particular,

$$
\varphi(1, \lambda), \varphi^{\prime}(1, \lambda), \vartheta(1, \lambda), \vartheta^{\prime}(1, \lambda) \rightarrow+\infty \quad \text { as } \quad \lambda \rightarrow-\infty .
$$

Note that $\left\{\mu_{n}\right\},\left\{\nu_{n}\right\},\left\{\tau_{n}\right\},\left\{\varrho_{n}\right\}$ are the zeros of $\varphi(1, \lambda), \vartheta^{\prime}(1, \lambda), \varphi^{\prime}(1, \lambda), \vartheta(1, \lambda)$ respectively.

2.2. The periodic case. We consider the 2-periodic operator $H_{\pi}=-\frac{d^{2}}{d x^{2}}+q_{\pi}(x)$ on $[0,2]$ given by (1.5). It is well known, that the spectrum of $H_{\pi}$ discrete and is eigenvalues $\lambda_{0}^{+}, \lambda_{n}^{ \pm}, n \geqslant$ 1 , which satisfy $\lambda_{n-1}^{+}<\lambda_{n}^{-} \leqslant \lambda_{n}^{+}, n \geqslant 1$. The sequence $\lambda_{0}^{+}<\lambda_{1}^{-} \leqslant \lambda_{1}^{+}<\ldots$ is the spectrum of equation

$$
-y^{\prime \prime}+q_{\pi} y=\lambda y
$$

with 2-periodic boundary conditions, i.e. $y(x+2)=y(x), x \in \mathbb{R}$. If $\lambda_{n}^{-}=\lambda_{n}^{+}$for some $n$, then this number $\lambda_{n}^{ \pm}$is a double eigenvalue of Eq. (2.5) with 2-periodic boundary conditions. The lowest eigenvalue $\lambda_{0}^{+}$is always simple, and the corresponding eigenfunction is 1-periodic. The 
eigenfunctions corresponding to the eigenvalue $\lambda_{n}^{ \pm}$are 1-periodic, when $n$ is even and they are antiperiodic, i.e. $y(x+1)=-y(x), \quad x \in \mathbb{R}$, when $n$ is odd. We introduce the Lyapunov function $\Delta=\frac{1}{2}\left(\varphi^{\prime}(1, \lambda)+\vartheta(1, \lambda)\right)$ It is well known that

$$
\Delta\left(\lambda_{0}^{+}\right)=1, \quad \Delta\left(\lambda_{n}^{ \pm}\right)=(-1)^{n}, \quad(-1)^{n} \Delta\left(\left[\lambda_{n}^{-}, \lambda_{n}^{+}\right]\right) \subset[1, \infty), n \geqslant 1 .
$$

2.3. Jost functions. We recall well-known result about the Jost function from [9], 23], 8]. The Schrödinger equation

$$
-f^{\prime \prime}+q(x) f=k^{2} f, \quad k \in \mathbb{C} \backslash\{0\},
$$

has unique solutions $f_{ \pm}(x, k)$ such that $f_{+}(x, k)=e^{i x k}$ for $x>1$ and $f_{-}(x, k)=e^{-i k x}$ for $x<0$. Outside the support of $q$ any solutions of (2.7) have to be combinations of $e^{ \pm i k x}$. The Wronskian $w$ for Case 3 is given by

$$
w(k)=\left\{f_{-}(\cdot, k), f_{+}(\cdot, k)\right\}=i k f_{+}(0, k)+f_{+}^{\prime}(0, k),
$$

where $\{f, g\}=f g^{\prime}-f^{\prime} g$. The functions $f_{+}(0, k), f_{+}^{\prime}(0, k), w(k)$ are entire, real on the imaginary line and satisfy as $|k| \rightarrow \infty, k \in \overline{\mathbb{C}}_{+}$:

$$
\begin{aligned}
& f_{+}(0, k)=1+O(1 / k), \quad f_{+}^{\prime}(0, k)=i k+O(1), \\
& w(k)=2 i k+O(1),
\end{aligned}
$$

uniformly in $\arg \in[0, \pi]$. Let $F$ be one of the functions $f_{+}(0, \cdot), f_{+}^{\prime}(0, \cdot)$ or $w$. The function $F$ has only finite simple number of zeros in the upper half-plane $\mathbb{C}_{+}$given by

$$
\begin{array}{ll}
\text { Case 1: } & i\left|E_{1}\right|^{\frac{1}{2}}, \ldots, i\left|E_{m}\right|^{\frac{1}{2}} \in i \mathbb{R}_{+}, \\
\text {Case 2 }: & i\left|\widetilde{E}_{1}\right|^{\frac{1}{2}}, \ldots, i\left|\widetilde{E}_{N}\right|^{\frac{1}{2}} \in i \mathbb{R}_{+}, \\
\text {Case } 3: & i\left|\mathcal{E}_{1}\right|^{\frac{1}{2}}, \ldots, i\left|\mathcal{E}_{\mathcal{N}}\right|^{\frac{1}{2}} \in i \mathbb{R}_{+},
\end{array}
$$

and an infinite number of zeros, so-called resonances, in $\mathbb{C}_{-}$and possibly one simple zero at 0 (for $q \neq 0$ ). By definition, a zero of $F$ is called a resonance of the corresponding Schrödinger operator. The multiplicity of the resonance is the multiplicity of the corresponding zero of $F$ and it can be any number, see [15]. Introduce the norm $\|u\|^{2}=\int_{0}^{1}|u(x)|^{2} d x$ in $L^{2}(0,1)$.

2.4. Proof Theorem 1.1, i-ii) Dirichlet and Neumann boundary conditions. Recall the identity from [15]:

$$
e^{-i k} f_{+}^{\prime}(0, k)=i k \vartheta(1, k)-\vartheta^{\prime}(1, k) .
$$

From (2.11), (2.4) and (1.6) we have that $\widetilde{E}_{1}>\nu_{0}$. Thus if $\nu_{0} \geqslant 0$, then the operator $\widetilde{T}$ has not eigenvalues. We fix a real potential $q_{0} \in L^{1}\left(\mathbb{R}_{+}\right)$such that $\operatorname{supp} q_{0} \in[0,1]$ and $\nu_{0}\left(q_{0}\right)=0$. Define the potential $q_{\varepsilon}, \varepsilon \in \mathbb{R}$ by

$$
\operatorname{supp} q_{\varepsilon} \subset[0,1], \quad q_{\varepsilon}(x)=q_{0}(x)-\varepsilon, \quad x \in[0,1] .
$$

We sometimes write $f_{+}(0, k, \varepsilon), \mu_{n}(\varepsilon),$. instead of $f_{+}(0, k), \mu_{n}, .$. when several potentials $q_{\varepsilon}$ are being dealt with. The operators in (1.3)-(1.4) and (1.1) with the potential $q_{\varepsilon}$ we denote by $H_{0}(\varepsilon), H_{1}(\varepsilon), \ldots$ and $T(\varepsilon), \ldots$ and the corresponding eigenvalues by $\mu_{n}(\varepsilon), \nu_{n}(\varepsilon) \ldots$ and $E_{n}(\varepsilon), \ldots$. Note that the eigenvalues $\mu_{n}(\varepsilon), \nu_{n}(\varepsilon), \ldots$ satisfy

$$
\mu_{n}(\varepsilon)=\mu_{n}(0)-\varepsilon, \quad \nu_{n}(\varepsilon)=\nu_{n}(0)-\varepsilon, \ldots \quad \tau_{n}(\varepsilon)=\tau_{n}(0)-\varepsilon, \quad \varrho_{n}(\varepsilon)=\varrho_{n}(0)-\varepsilon .
$$


Firstly we consider the operator $\widetilde{T}$ with the Neumann boundary condition. Due to (2.11) the Jost function for $\widetilde{T}(\varepsilon)$ for $k=i t, t \in \mathbb{R}$ satisfies

$$
e^{-i k} f_{+}^{\prime}(0, k, \varepsilon)=-\vartheta^{\prime}\left(1, k^{2}, \varepsilon\right)+i k \vartheta\left(1, k^{2}, \varepsilon\right)=i k \vartheta\left(1, \varepsilon-t^{2}, 0\right)-\vartheta^{\prime}\left(1, \varepsilon-t^{2}, 0\right) .
$$

Define functions

$$
F(t, \varepsilon)=-f\left(t^{2}, \varepsilon\right)-t g\left(t^{2}, \varepsilon\right), \quad f\left(t^{2}, \varepsilon\right)=\vartheta^{\prime}\left(1, \varepsilon-t^{2}, q_{0}\right), \quad g\left(t^{2}, \varepsilon\right)=\vartheta\left(1, \varepsilon-t^{2}, q_{0}\right) .
$$

These functions are entire in $t, \varepsilon$ and satisfy

$$
f(0,0)=0, \quad g(0,0) \neq 0, \quad F(0,0)=0, \quad F_{t}(0,0) \neq 0 .
$$

Then due to the Implicit Function Theorem there exists a function $t(\varepsilon)$, analytic in small disk $\{|\varepsilon|<\tau\}$ such that $F(t(\varepsilon), \varepsilon)=0$ in the disk $\{|\varepsilon|<\tau\}$ (and here $\widetilde{E}_{1}(\varepsilon)=-t^{2}(\varepsilon)$ for $\varepsilon>0$ ).

We have $t(\varepsilon)=t_{1} \varepsilon+O\left(\varepsilon^{2}\right)$. Then from (2.13) we obtain for $t=t(\varepsilon)$ as $\varepsilon \rightarrow 0$ :

$$
\begin{array}{r}
\vartheta^{\prime}\left(1, \varepsilon-t^{2}, 0\right)=-t(\varepsilon) \vartheta\left(1, \varepsilon-t^{2}, 0\right)=t_{1} \varepsilon \vartheta(1,0,0)+O\left(\varepsilon^{2}\right), \\
\vartheta^{\prime}\left(1, \varepsilon-t^{2}, 0\right)=\varepsilon \dot{\vartheta}^{\prime}(1,0,0)+O\left(\varepsilon^{2}\right),
\end{array}
$$

which yields $t_{1}=-\frac{\dot{\vartheta}^{\prime}(1,0,0)}{\vartheta(1,0,0)}>0$, where $\dot{u}=\frac{\partial}{\partial \lambda} u$.

If $\varepsilon>0$ is small enough, then $\nu_{0}(\varepsilon)=-\varepsilon$ and $\varrho_{1}(\varepsilon)=\varrho_{1}\left(q_{0}\right)-\varepsilon>0$, since there is the basic relation (1.6). Thus we obtain $\nu_{0}(\varepsilon)<\widetilde{E}_{1}(\varepsilon)<0<\varrho_{1}(\varepsilon)$. If $\varepsilon$ is increasing then all eigenvalues $\nu_{0}(\varepsilon)<\widetilde{E}_{1}(\varepsilon)<0<\varrho_{1}(\varepsilon)$ move monotonically to left and at $\varepsilon_{1}=\tau_{1}(0)$ we have $\tau_{1}\left(\varepsilon_{1}\right)=0$.

Secondly, we consider the operator $T$ with the Dirichlet boundary condition. The function $f_{+}(0, k)$ is expressed in terms of the fundamental solutions $\varphi, \vartheta$ by

$$
e^{-i k} f_{+}(0, k)=\varphi^{\prime}\left(1, k^{2}\right)-i k \varphi\left(1, k^{2}\right) \quad \forall k \in \mathbb{C} .
$$

Note that if the operator $H_{01}$ have eigenvalue $\tau_{1} \geqslant 0$, then from (2.16), (2.4) and (1.6) we deduce that the operator $T$ has not any eigenvalue. Let $\varepsilon=\varepsilon_{1}+z$, where $\varepsilon_{1}=\tau_{1}(0)$. Then due to (2.16) the Jost function $f_{+}(0, k, \varepsilon)$ for the operator $T(\varepsilon)$ with satisfies at $k=i t, t \in \mathbb{R}$ :

$$
e^{-i k} f_{+}(0, k, \varepsilon)=\varphi^{\prime}\left(1, z-t^{2}, \varepsilon_{1}\right)+t \varphi\left(1, z-t^{2}, \varepsilon_{1}\right) .
$$

We rewrite the rhs of the last identity in the form:

$$
F_{o}(t, \varepsilon)=f_{o}\left(t^{2}, \varepsilon\right)+t g_{o}\left(t^{2}, \varepsilon\right), \quad f_{o}\left(t^{2}, z\right)=\varphi^{\prime}\left(1, z-t^{2}, \varepsilon_{1}\right), \quad g_{o}\left(t^{2}, z\right)=\varphi\left(1, z-t^{2}, \varepsilon_{1}\right) \text {. }
$$

These functions are entire in $t, z$ and satisfy

$$
f_{o}(0,0)=0, \quad g_{o}(0,0) \neq 0, \quad F_{o}(0,0)=0, \quad \frac{\partial}{\partial t} F_{o}(0,0) \neq 0 .
$$

Then the Implicit Function Theorem gives that there exists a function $t_{o}(z)$, analytic in small disk $\{|z|<\delta\}$ such that $F\left(t_{o}(z), z\right)=0$ in the disk $\{|z|<\delta\}$. Here we have $E_{1}(\varepsilon)=t_{o}^{2}(z), z=$ $\varepsilon-\varepsilon_{1}$. We have $t(z)=t_{1} z+O\left(z^{2}\right)$ as $z \rightarrow 0$. Then from $f_{+}(0, k, \varepsilon)=0$ and (2.17) we obtain

$$
\begin{array}{r}
\varphi^{\prime}\left(1, z-t^{2}, \varepsilon_{1}\right)=-t(z) \varphi\left(1, z-t^{2}, \varepsilon_{1}\right)=-t_{1} z \varphi\left(1,0, \varepsilon_{1}\right)+O\left(z^{2}\right), \\
\varphi^{\prime}\left(1, z-t^{2}, \varepsilon_{1}\right)=\dot{\varphi}^{\prime}\left(1,0, \varepsilon_{1}\right) z+O\left(z^{2}\right),
\end{array}
$$

which yields $t_{1}=-\frac{\dot{\varphi}^{\prime}\left(1,0, \varepsilon_{1}\right)}{\varphi\left(1,0, \varepsilon_{1}\right)}>0$, since $\varphi\left(1, \lambda, \varepsilon_{1}\right) \rightarrow \infty$ as $\lambda \rightarrow-\infty$ and $\mu_{1}>0$. 
If $z>0$ is small enough, then $\tau_{1}(\varepsilon)=-z$ and $\mu_{1}(\varepsilon)=\mu_{1}(0)-\varepsilon>0$, since there is the basic relation (1.6). Thus we obtain

$$
\tau_{1}(\varepsilon)<E_{1}(\varepsilon)<0<\mu_{1}(\varepsilon) \text {, and } \widetilde{E}_{1}(\varepsilon)<E_{1}(\varepsilon) .
$$

It is important that $\widetilde{E}_{1}(\varepsilon)<E_{1}(\varepsilon)$ for any $\varepsilon$ since $\sigma_{d}(T) \cap \sigma_{d}(\widetilde{T})=\emptyset$. If $\varepsilon$ is increasing then all eigenvalues $\tau_{1}(\varepsilon)<E_{1}(\varepsilon)<\mu_{1}(\varepsilon)$ and $\widetilde{E}_{1}(\varepsilon)<E_{1}(\varepsilon)$ move monotonically to left. If $\varepsilon$ is increasing more, then we have $\mu_{1}\left(\varepsilon_{1}\right)=0$ at $\varepsilon_{1}=\mu_{1}(0)$, but this eigenvalues does not "create" eigenvalues for the operators $T(\varepsilon), \widetilde{T}(\varepsilon)$. If $\varepsilon$ is increasing again then we have $\nu_{1}\left(\varepsilon_{2}\right)=0$ at $\varepsilon_{2}=\nu_{1}\left(q_{0}\right)$. Using the above arguments for the case $\nu_{0}=0$ we obtain (1.7) and (1.8), and so on. Repeating these arguments we obtain (1.7)-(1.11), since we can take any $q_{0}$ and $\varepsilon$.

iii) We consider the Schrödiger operator $\mathcal{T}$ on the real line. Note that if the entire function $f_{+}^{\prime}(0, k)<0$ for $k \in i \mathbb{R}_{+}$and has the zero $k=0$, then using $w=i k f_{+}(0, \cdot)+f_{+}^{\prime}(0, \cdot)$ from (2.8) we deduce that the operator $\mathcal{T}$ has not any eigenvalue.

Recall that $q_{0} \in L^{1}\left(\mathbb{R}_{+}\right)$is such that $\operatorname{supp} q_{0} \in[0,1]$ and $\nu_{0}\left(q_{0}\right)=0$. We need the identity from [17]:

$$
e^{-i k} w(k)=2 i k \Delta(\lambda)+\lambda \varphi(1, \lambda)-\vartheta^{\prime}(1, \lambda) .
$$

Due to (2.20) the Wronskian for $q_{\varepsilon}$ satisfies at $k=i t$ :

$$
\begin{array}{r}
e^{-i k} w(k, \varepsilon)=2 i k \Delta(\lambda, \varepsilon)+\lambda \varphi(1, \lambda, \varepsilon)-\vartheta^{\prime}(1, \lambda, \varepsilon), \\
e^{t} w(i t, \varepsilon)=-2 t \Delta\left(\varepsilon-t^{2}, 0\right)-t^{2} \varphi\left(1, \varepsilon-t^{2}, 0\right)-\vartheta^{\prime}\left(1, \varepsilon-t^{2}, 0\right) .
\end{array}
$$

We rewrite the rhs of the last identity for $k=i t, t \in \mathbb{R}$ in the form:

$$
F=-f-g, f(t, \varepsilon)=\vartheta^{\prime}\left(1, \varepsilon-t^{2}, 0\right), g(t, \varepsilon)=2 t \Delta\left(\varepsilon-t^{2}, 0\right)+t^{2} \varphi\left(1, \varepsilon-t^{2}, 0\right) .
$$

These functions are entire in $t, \varepsilon$ and satisfy at $\varepsilon=t=0$ :

$$
f(0,0)=0, f_{t}(0,0)=0, g(0,0)=0, \quad g_{t}(0,0)=2 \Delta(0,0) \geqslant 2,
$$

since $\Delta(\lambda, 0) \geqslant 1$ for any $\lambda \leqslant \nu_{0}\left(q_{0}\right)$. We show that exists exactly one eigenvalue for small $\varepsilon>0$. From (2.20) we have the simple fact: $w(0,0)=0$ iff $\vartheta^{\prime}(1,0,0)=0$. Then the Implicit Function Theorem gives that there exists a function $t_{o}(\varepsilon)$, analytic in small disk $\{|\varepsilon|<\delta\}$ such that $F\left(t_{o}(\varepsilon), \varepsilon\right)=0$ in the disk $\{|\varepsilon|<\delta\}$. Moreover, from (2.21) we have $t_{o}(\varepsilon)=-C \varepsilon+O\left(\varepsilon^{2}\right)$, where $C=\frac{\dot{\vartheta}^{\prime}(1,0,0)}{2 \Delta(0,0)}<0$. Here we have $\mathcal{E}_{1}(\varepsilon)=-t_{o}^{2}(\varepsilon)$. Thus the eigenvalues $\nu_{0}(\varepsilon)$ at $\varepsilon=0$ creates two eigenvalues $\nu_{0}(\varepsilon)<\widetilde{E}_{1}(\varepsilon)<\mathcal{E}_{1}(\varepsilon)<0$ for small $\varepsilon>0$. If $\varepsilon$ is increasing then repeating the arguments for the case of the operator $\widetilde{T}$ we have (1.12), (1.13). Here we need the fact: if $\mathcal{E}$ is an eigenvalue of $\mathcal{T}$, then $\mathcal{E} \notin \sigma_{d}(T) \cup \sigma_{d}(\widetilde{T})$.

Proof of Corollary 1.2. The Schrödinger equation $-f^{\prime \prime}+q_{e}(x) f=k^{2} f, \quad k \in \mathbb{C} \backslash\{0\}$, has the Jost solutions $\psi_{ \pm}(x, k)$ such that $\psi_{+}(x, k)=e^{i x k}, x \geqslant 1$ and $\psi_{-}(x, k)=e^{-i k x}, \quad x \leqslant-1$. Note that the symmetry of the potential $q_{e}$ yields

$$
\psi_{+}(x, k)=f_{+}(x, k)=\psi_{-}(-x, k) \quad \forall x \in[0,1] .
$$

This implies that the Wronskian $w_{e}(k)$ for the potential $q_{e}$ satisfies

$$
w_{e}(k)=\left.\left\{\psi_{+}(x, k), \psi_{-}(x, k)\right\}\right|_{x=0}=2 f_{+}(0, k) f_{+}^{\prime}(0, k),
$$

where $f_{+}(0, k)$ and $f_{+}^{\prime}(0, k)$ are the Jost functions for the Cases 1 and 2 respectively with the potential $q$. This identity and Theorem 1.1 yield the proof of Corollary 1.2,

In order to discuss resonances we define the class of all Jost functions from [15]: 
Definition J. By $\mathcal{J}$ we mean the class of all entire functions $f$ having the form

$$
f(k)=1+\frac{\hat{F}(k)-\hat{F}(0)}{2 i k}, \quad k \in \mathbb{C},
$$

where $\hat{F}(k)=\int_{0}^{1} F(x) e^{2 i x k} d x$ is the Fourier transformation of $F \in \mathcal{P}$ and a set of zeros $K=\left\{k_{n}, n \in \mathbb{N}\right\}$ (counted with multiplicity) of $f$ satisfy:

1) The set $K=\bar{K}$ and has not zeros from $\mathbb{R} \backslash\{0\}$ and has possibly one simple zero at 0 .

2) Let $\# E$ be the number of zeros of $K$ on the set $E \subset \mathbb{C}$. The set $K$ has finite number elements $k_{1}, . ., k_{m}$ from $\mathbb{C}_{+}$, which are simple, belong to $i \mathbb{R}_{+}$and if they are labeled by $\left|k_{1}\right|>$ $\left|k_{2}\right|>\ldots>\left|k_{m}\right|>0$, then intervals on $i \mathbb{R}_{-}$defined $I_{j}=\left(-k_{j},-k_{j+1}\right), j \in \mathbb{N}_{m-1}$ and $I_{m}=$ $\left(-k_{m}, 0\right]$ satisfy

$$
-k_{j} \notin K, \quad j \in \mathbb{N}_{m}:=\{1,2, \ldots, m\}, \quad\left\{\begin{array}{l}
\# I_{j} \geqslant 1 \text { is odd, } j \in \mathbb{N}_{m-1} \\
\# I_{m} \geqslant 0 \text { is even }
\end{array} .\right.
$$

We recall the basic result about inverse problem (including the characterization) for compactly supported potentials from [15].

Theorem 2.2. The mapping $\psi: \mathcal{P} \rightarrow \mathcal{J}$ given by $q \rightarrow \psi$ is one-to-one and onto, where $\psi(k)=f_{+}(0, k)$.

Thus if $q \in \mathcal{P}$, then $\psi \in \mathcal{J}$. Conversely, for each $f \in \mathcal{J}$ exists a unique potential $q \in \mathcal{P}$ such that the Jost function $\psi=f$. In fact using this result we reformulate the problem for the differential operator as the problem of the entire function theory. We need Theorem 3 from [16]:

Theorem 2.3. Let $\psi^{o} \in \mathcal{J}$ have zeros $\left\{k_{n}^{o}\right\}_{1}^{\infty}$ and let the sequence complex points $\left\{k_{n}\right\}_{1}^{\infty}$ satisfy 1) and 2) in Condition $J$ and $\sum_{n \geqslant 1} n^{3}\left|k_{n}-k_{n}^{o}\right|^{2}<\infty$. Then $\left\{k_{n}\right\}_{1}^{\infty}$ is a sequence of zeroes of unique $\psi \in \mathcal{J}$, which is the Jost function for unique $q \in \mathcal{P}$.

Proof of Theorem 1.3, i) Let a resonance $k_{o}=-i r \in I_{j}$. Then from (2.16) we obtain $\varphi^{\prime}\left(1, k_{o}^{2}\right)=r \varphi\left(1, k_{o}^{2}\right)$, which yields $\operatorname{sign} \varphi^{\prime}\left(1, k_{o}^{2}\right)=\operatorname{sign} \varphi\left(1, k_{o}^{2}\right)$. From (1.8) we deduce that $\mu_{j}<r_{o}^{2}<\tau_{j+1}$, since $\varphi^{\prime}(1, \lambda) \rightarrow+\infty$ and $\varphi(1, \lambda) \rightarrow+\infty$ as $\lambda \rightarrow-\infty$.

ii) We take any potential $q^{o} \in \mathcal{P}$ such that the Jost function $\psi^{o} \in \mathcal{P}$ has zeros $\left\{k_{n}^{o}\right\}_{1}^{\infty}$ only from $\mathbb{C}_{-}$. We take the half-disc $\mathcal{D}_{r}:=\left\{k \in \mathbb{C}_{-}:|k|<r\right\}$ for a radius $r>3+\left|p_{1}\right|$ large enough such that the number $N_{r}$ of zeros $\left\{k_{n}^{o}\right\}$ in $\mathcal{D}_{r}$ satisfy $N_{r}>m+\left(s_{1}+. .+s_{m}\right)$, since we have the following result from [27]: $N_{r}=\frac{r}{2 \pi}(1+o(1)$ ar $r \rightarrow \infty$. We construct the new sequence of zeros $\left\{k_{n}\right\}$ :

- if $k_{n}^{o} \notin \mathcal{D}_{r}$, then $k_{n}=k_{n}^{o}$.

- Consider $N_{r}$ zeros $\left\{k_{n}^{o}\right\}$ in $\mathcal{D}_{r}$. We have three cases:

a) We remove $m$ zeros from $\mathbb{D}_{-}(r)$ on the points $k_{j}=p_{j} \in i \mathbb{R}_{+}$for each $j \in \mathbb{N}_{m}$.

b) We remove $s_{j}$ zeros from $\mathbb{D}_{-}(r)$ on each interval $I_{j}=\left(-k_{j},-k_{j+1}\right), j \in \mathbb{N}_{m-1}$ and $I_{m}=\left(-k_{m}, 0\right]$ at $j=m$.

c) We remove all remaining zeros $n_{r}=N_{r}-m-\left(s_{1}+. .+s_{m}\right)>0$ from $\mathbb{D}_{-}(r)$ on point $-p_{1}-i$ with the multiplicity $n_{r}$. Recall that $r$ is large enough and $r>3+\left|p_{1}\right|$.

Thus the sequence $\left\{k_{n}\right\}$ satisfy 1) and 2) in Condition J. Then due to Theorem $2.3\left(k_{n}\right)_{1}^{\infty}$ is a sequence of zeroes of unique $f \in \mathcal{J}$, which is the Jost function for unique $q \in \mathcal{P}$. 
Proof of Corollary [1.4, i) We need an inequality from [11], [26]: if $q \in L^{1}(\mathbb{R})$, then

$$
\sum_{\mathcal{E}_{n}<0}\left|\mathcal{E}_{n}\right|^{\frac{1}{2}} \leqslant \frac{1}{2} \int_{\mathbb{R}} q_{-}(x) d x .
$$

From Theorem 1.1 and inequalities (1.19), (1.20) and (2.26) we obtain (1.22), (1.24) and (1.23) for $\mathfrak{S}=\sum_{\mu_{n}<0}\left|\mu_{n}\right|^{\frac{1}{2}}$. Applying (2.26) to the operator $\mathcal{T}_{e}$ and estimates from (1.6), Corollary $1.2\left(\left|\lambda_{n}^{ \pm}\right| \leqslant\left|\mathcal{E}_{n}^{e}\right|\right.$ for negative eigenvalues) we obtain

$$
\sum_{n>0, \lambda_{n}^{ \pm}<0}\left|\lambda_{n}^{ \pm}\right|^{\frac{1}{2}}<\sum_{\mathcal{E}_{n}^{e}<0}\left|\mathcal{E}_{n}^{e}\right|^{\frac{1}{2}} \leqslant \frac{1}{2} \int_{\mathbb{R}} q_{e-}(x) d x=\int_{0}^{1} q_{-}(x) d x .
$$

ii) Substituting the estimate $\|q\| \leqslant 2 \gamma \max \left\{1, \gamma^{\frac{1}{3}}\right\}$ from (1.21) into (1.22), (1.23) we obtain (1.25).

We now illustrate our results

Example i) For any number $c<0$ there exists a potential $q \in \mathcal{P}$ such that the operator $T_{q}$ has not eigenvalues and $\int_{0}^{1} q(x) d x=c$.

ii) Let $c>0$ and let $I_{j}=\left(t_{j}, m_{j}\right) \subset \mathbb{R}_{-}, j \in \mathbb{N}_{m}$ be finite sequence of intervals such that $t_{1}<m_{1}<t_{2}<m_{2}<\ldots<t_{m}<m_{m}<0$. Then there exists a potential $q \in \mathcal{P}$ such that $\int_{0}^{1} q(x) d x=c$ and the operator $T_{q}$ has an eigenvalue $E_{j} \in I_{j}$ for any $j \in \mathbb{N}_{m}$. There are no other eigenvalues.

Proof. i) Let $c<0$. We define two strongly increasing sequences by

$$
m_{j}=\left\{\begin{array}{ll}
(\pi j)^{2}, & j \in \mathbb{N}_{n} \\
(\pi j)^{2}+c, & j>n
\end{array}, \quad t_{j}= \begin{cases}\pi^{2}\left(j-\frac{1}{2}\right)^{2}, & j \in \mathbb{N}_{n}, \\
\pi^{2}\left(j-\frac{1}{2}\right)^{2}+c, & j>n\end{cases}\right.
$$

for some $n>|c|$. They satisfy $t_{j}<m_{j}<t_{j+1}<\ldots j \in \mathbb{N}$. Due to result of [23] there exists a potential $q \in L^{2}(0,1)$ with $\int_{0}^{1} q(x) d x=c$ and $\mu_{j}(q)=m_{j}$ and $\tau_{j}(q)=t_{j}$ for all $j \geqslant 1$. Then due to (1.8) the operator $T=-\frac{d^{2}}{d x^{2}}+q$ on $\mathbb{R}_{+}$has not eigenvalues.

ii) Let $c>0$. Let $t_{j}=\pi^{2}\left(j-\frac{1}{2}\right)^{2}+c, j>m$ and $m_{j}=(\pi j)^{2}+c, j>m$. Thus the numbers $t_{j}, m_{j}, j \in \mathbb{N}$ satisfy $t_{j}<m_{j}<t_{j+1}$ for all $j \in \mathbb{N}$. Due to result of [23] there exists a potential $q \in L^{2}(0,1)$ with $\int_{0}^{1} q(x) d x=c$ and $\mu_{j}(q)=m_{j}$ and $\tau_{j}(q)=t_{j}$ for all $j \geqslant 1$. Then due to (1.8) the operator $T=-\frac{d^{2}}{d x^{2}}+q$ on $\mathbb{R}_{+}$has an eigenvalue $E_{j} \in I_{j}$ for any $j \in \mathbb{N}_{m}$ and there are no other eigenvalues.

Acknowledgments. This work was supported by the RSF grant No. 18-11-00032.

\section{REFERENCES}

[1] Albeverio, S.; Kostenko, A.; Malamud, M. Spectral theory of semibounded Sturm-Liouville operators with local interactions on a discrete set. J. Math. Phys. 51 (2010), no. 10, 102102, 24 pp.

[2] Albeverio, S.; Kostenko, A.; Malamud, M.; Neidhardt, H. Spherical Schrödinger operators with d-type interactions. J. Math. Phys. 54 (2013), no. 5, 052103, 24 pp.

[3] Bargmann, V. On the number of bound states in a central field of force. Proc. Nat.Acad. Sci. U.S.A. 38 (1952), 961-966.

[4] Calogero, V. Upper and lower limits for the number of bound states in a given central potential. Comm. Math. Phys. 1 (1965), 80-88.

[5] Cohn J. H. E. On the number of negative eigen-values of a singular boundary value problem. J. London Math. Soc. 40 (1965), 523-525. 
[6] Dahlberg, B.; Trubowitz, E. The inverse Sturm-Liouville problem. III. Comm. Pure Appl. Math. $37(1984)$, no. 2, 255-267.

[7] Damanik, D.; Hundertmark, D.; Simon, B. Bound states and the Szegö condition for Jacobi matrices and Schrödinger operators. J. Funct. Anal. 205 (2003), no. 2, 357-379.

[8] Deift, P.; Trubowitz, E. Inverse scattering on the line. Communications on Pure and Applied Mathematics, 32(2) (1979), 121-251.

[9] Faddeev, L. The inverse problem in the quantum theory of scattering. J. Math. Phys. 4,1(1963), 72-104, translated from Usp. Mat. Nauk (1959).

[10] Garnett, J.; Trubowitz, E. Gaps and bands of one dimensional periodic Schrödinger operators II. Comment. Math. Helv. 62(1987), 18-37.

[11] Hundertmark, D.; Lieb, E.; Thomas, L. A sharp bound for an eigenvalue moment of the one-dimensional Schrdinger operator. Adv. Theor. Math. Phys. 2 (1998), no. 4, 719-731.

[12] Isaacson, E. L.; Trubowitz, E. The inverse Sturm-Liouville problem. I. Comm. Pure Appl. Math. 36 (1983), no. 6, 767-783.

[13] Isaacson, E. L.; McKean, H. P.; Trubowitz, E. The inverse Sturm-Liouville problem. II. Comm. Pure Appl. Math. 37 (1984), no. 1, 1-11.

[14] Jost, R.; Kohn, W. On the relation between phase shift energy levels and the potential. Danske Vid. Selsk. Mat.-Fys. Medd. 27, (1953). no. 9, 19 pp.

[15] Korotyaev, E. Inverse resonance scattering on the half line. Asymptot. Anal. 37 (2004), no. 3-4, $215-226$.

[16] Korotyaev, E. Stability for inverse resonance problem. Int. Math. Res. Not. 2004, no. 73, 3927-3936.

[17] Korotyaev, E. Inverse resonance scattering on the real line. Inverse Problems 21(2005), no. 1, 325-341.

[18] Korotyaev, E. The inverse problem and trace formula for the Hill operator, II. Math. Z. 231(1999), $345-368$

[19] Korotyaev, E. Estimate of periodic potentials in terms of gap lengths. Commun. Math. Phys. 197(1998), $521-526$.

[20] Korotyaev, E. Estimates for the Hill operator. I. J. Differential Equations 162 (2000), no. 1, 1-26.

[21] Gelfand-Levitan equation and Schrödinger operators with compactly supported potentials

[22] Korotyaev, E.; Chelkak, D. The inverse Sturm-Liouville problem with mixed boundary conditions, St. Petersburg Math. Journal, 21(2009), no 5, 114-137.

[23] Marchenko, V. Sturm-Liouville operator and applications. Basel, Birkhäuser 1986.

[24] Pöschel, P.; Trubowitz E. Inverse Spectral Theory. Boston, Academic Press, 1987.

[25] Solomyak, M. On a class of spectral problems on the half-line and their applications to multi-dimensional problems. J. Spectr. Theory 3 (2013), no. 2, 215-235.

[26] Weidl, T. On the Lieb-Thirring constants $L_{\gamma, 1}$ for $\gamma \geqslant \frac{1}{2}$. Comm. Math. Phys. 178 (1996), no. 1, $135-146$.

[27] Zworski, M. Distribution of poles for scattering on the real line, J. Funct. Anal. 73(1987), 277-296.

Department of Math. Analysis, Saint-Petersburg State University, Universitetskaya nab. 7/9, St. Petersburg, 199034, Russia, Korotyaev@Gmail.com, E.Korotyaev@spbu.ru 